See discussions, stats, and author profiles for this publication at: https://www.researchgate.net/publication/284020935

\title{
Effectiveness and safety of cyclosporine in pediatric plaque psoriasis: A multicentric retrospective analysis
}

Article in Journal of Dermatological Treatment · November 2015

DOl: 10.3109/09546634.2015.1120852

\section{CITATIONS}

28

13 authors, including:

Vito Di Lernia

Azienda Unità Sanitaria Locale Reggio Emilia

165 PUBLICATIONS 2,692 CITATIONS

SEE PROFILE

Valeria Boccaletti

University Hospital of Brescia

36 PUBLICATIONS 498 CITATIONS

SEE PROFILE

Some of the authors of this publication are also working on these related projects:

Sanitary Entomology View project

Dermatology and Infectious Diseases View project
Stingeni Luca

Università degli Studi di Perugia 225 PUBLICATIONS 1,556 CITATIONS

SEE PROFILE

Claudio Guarneri

Università degli Studi di Messina

332 PUBLICATIONS 1,826 CITATIONS

SEE PROFILE 


\section{Effectiveness and safety of cyclosporine in pediatric plaque psoriasis: a multicentric retrospective analysis.}

V. Di Lernia, L. Stingeni, V. Boccaletti, P.G. Calzavara Pinton, C. Guarneri, A. Belloni Fortina, M. Panzone, M. Corazza, I. Neri, S. Cambiaghi, C. Lasagni, E. Ficarelli \& P. Gisondi

To cite this article: V. Di Lernia, L. Stingeni, V. Boccaletti, P.G. Calzavara Pinton, C. Guarneri, A. Belloni Fortina, M. Panzone, M. Corazza, I. Neri, S. Cambiaghi, C. Lasagni, E. Ficarelli \& P. Gisondi (2015): Effectiveness and safety of cyclosporine in pediatric plaque psoriasis: a multicentric retrospective analysis., Journal of Dermatological Treatment, DOI: 10.3109/09546634.2015.1120852

To link to this article: http://dx.doi.org/10.3109/09546634.2015.1120852

Accepted author version posted online: 16 Nov 2015.

Submit your article to this journal

Џ Article views: 8

View related articles

View Crossmark data \lceil 


\section{Effectiveness and safety of cyclosporine in pediatric plaque psoriasis: a multicentric retrospective analysis.}

V. Di Lernia, L. Stingeni, V. Boccaletti, P.G. Calzavara Pinton, C. Guarneri, A. Belloni Fortina, M. Panzone, M. Corazza, I. Neri, S. Cambiaghi, C. Lasagni, E. Ficarelli, P. Gisondi

doi: 10.3109/09546634.2015.1120852

\section{Abstract}

Cyclosporine (CysA) is effective for psoriasis in adult patients but little data exist about its efficacy and safety in childhood and adolescence psoriasis.

Objectives. To assess the effectiveness and safety of CysA for childhood and adolescence psoriasis.

Methods. Retrospective analysis of a group of children and adolescents (age $<17$ years) with plaque psoriasis treated with CysA at several Italian dermatology clinics.

Results. Our study population consisted of 38 patients. The median age at the start of treatment was 12,3 years. Therapy duration varied from 1 to 36 months. The median maintenance dosage per day was $3,2 \mathrm{mg} / \mathrm{kg}$ (range $2-5 \mathrm{mg} / \mathrm{kg}$ ). Fifteen patients $(39,4 \%)$ achieved a complete clearance or a good improvement of their psoriasis defined by an improvement from baseline of $\geq 75 \%$ in the Psoriasis Area and Severity Index at week 16 . Eight patients $(21.05 \%)$ discontinued the treatment due to laboratory anomalies or adverse events. Serious events were not recorded.

Conclusions. In this case series CysA was effective and well tolerated treatment in a significant quote of children. CysA, when carefully monitored, may represent a therapeutic alternative to the currently used systemic immunosuppressive agents for severe childhood psoriasis.

(C) 2015 Taylor \& Francis. This provisional PDF corresponds to the article as it appeared upon acceptance. Fully formatted PDF and full text (HTML) versions will be made available soon.

DISCLAIMER: The ideas and opinions expressed in the journal's Just Accepted articles do not necessarily reflect those of Taylor \& Francis (the Publisher), the Editors or the journal. The Publisher does not assume any responsibility for any injury and/or damage to persons or property arising from or related to any use of the material contained in these articles. The reader is advised to check the appropriate medical literature and the product information currently provided by the manufacturer of each drug to be administered to verify the dosages, the method and duration of administration, and contraindications. It is the responsibility of the treating physician or other health care professional, relying on his or her independent experience and knowledge of the patient, to determine drug dosages and the best treatment for the patient. Just Accepted articles have undergone full scientific review but none of the additional editorial preparation, such as copyediting, typesetting, and proofreading, as have articles published in the traditional manner. There may, therefore, be errors in Just Accepted articles that will be corrected in the final print and final online version of the article. Any use of the Just Accepted articles is subject to the express understanding that the papers have not yet gone through the full quality control process prior to publication. 


\section{Effectiveness and safety of cyclosporine in pediatric plaque psoriasis: a multicentric retrospective}

analysis.

Running Head: Cyclosporine in pediatric plaque psoriasis

Word count: 2176; Table count: 1

Key words: psoriasis, childhood, cyclosporine, therapy, treatment.

V. Di Lernia ${ }^{1}$, L. Stingeni ${ }^{2}$, V. Boccaletti ${ }^{3}$, P.G. Calzavara Pinton ${ }^{4}$, C. Guarneri ${ }^{5}$, A. Belloni Fortina ${ }^{6}$, M. Panzone $^{7}$, M. Corazza ${ }^{8}$, I. Neri ${ }^{9}$, S. Cambiaghi ${ }^{10}$, C. Lasagni ${ }^{11}$, E. Ficarelli ${ }^{1}$, P. Gisondi ${ }^{12}$.

${ }^{1}$ Dermatology Unit, Arcispedale Santa Maria Nuova IRCCS, Reggio Emilia, ${ }^{2}$ Section of Clinical, Allergological and Venereological Dermatology, Department of Medicine, University of Perugia, ${ }^{3}$ Section of Dermatology, Department of Clinical and Experimental Medicine, Parma University Hospital, ${ }^{4}$ Department of Dermatology, University of Brescia, ${ }^{5}$ Department of Clinical and Experimental Medicine, Section of Dermatology, University of Messina, ${ }^{6}$ Pediatric Dermatology Unit, Department of Medicine, University of Padua, ${ }^{7}$ Department of Dermatology, University of Turin, ${ }^{8}$ Department of Medical Sciences, Section of Dermatology, University of Ferrara, ${ }^{9}$ Dermatology Unit, Department of Specialistic, Diagnostic and Experimental Medicine, University of Bologna, S. Orsola-Malpighi Hospital, Bologna, Italy, ${ }^{10}$ UOC Dermatologia Pediatrica, Ospedale Maggiore, Policlinic of Milan, ${ }^{11}$ Dermatology Unit, Department of Head and Neck Surgery, University of Modena, ${ }^{12}$ Department of Medicine, Section of Dermatology and Venereology, University of Verona, Italy.

Correspondence: V. Di Lernia, M.D., Dermatology Unit, Arcispedale Santa Maria Nuova-IRCCS, viale Risorgimento 68, 42123 Reggio Emilia, Italy. Email: vito.dilernia@asmn.re.it Fax: +390522 295382

\section{Funding sources: none}

Financial disclosure: V. Di Lernia served as Advisory Board Member for AbbVie, C. Guarneri received honoraria from lectures and board membership from Pfizer, C. Lasagni received honoraria from Abbott, Pfizer, and Janssen, P. Gisondi has been a consultant and/or speaker for Abbott, Janssen, Leo-Pharma, Lilly, Merck Sharp \& Dohme, Novartis, and Pfizer. 


\section{Summary}

Cyclosporine (CysA) is effective for psoriasis in adult patients but little data exist about its efficacy and safety in childhood and adolescence psoriasis.

Objectives. To assess the effectiveness and safety of CysA for childhood and adolescence psoriasis.

Methods. Retrospective analysis of a group of children and adolescents (age $<17$ years) with plaque psoriasis treated with CysA at several Italian dermatology clinics.

Results. Our study population consisted of 38 patients. The median age at the start of treatment was 12,3 years. Therapy duration varied from 1 to 36 months. The median maintenance dosage per day was $3,2 \mathrm{mg} / \mathrm{kg}$ (range $2-5 \mathrm{mg} / \mathrm{kg}$ ). Fifteen patients $(39,4 \%)$ achieved a complete clearance or a good improvement of their psoriasis defined by an improvement from baseline of $\geq 75 \%$ in the Psoriasis Area and Severity Index at week 16 . Eight patients (21.05\%) discontinued the treatment due to laboratory anomalies or adverse events. Serious events were not recorded.

Conclusions. In this case series CysA was effective and well tolerated treatment in a significant quote of children. CysA, when carefully monitored, may represent a therapeutic alternative to the currently used systemic immunosuppressive agents for severe childhood psoriasis. 


\section{Introduction}

Psoriasis is a common inflammatory skin disease that starts approximately in one third of patients in the first two decades of life [1-2]. Prevalence of childhood psoriasis is unknown, but it has been calculated to range from 0.1 to $3 \%$ in various parts of the world [2-3]. Psoriasis incidence may be increasing over time [4]. In children, psoriasis usually both follows a benign course and is often successfully managed with topical agents. However moderate or severe forms of the disease may be unresponsive to topical therapy and require more aggressive approaches, including phototherapy and pharmacologic interventions with systemic drugs. Most of systemic therapies have not been systematically investigated in moderate to severe childhood plaque psoriasis. Therefore potential beneficial effects of systemic drugs have been assumed on the basis of clinical evidences or experiences in adults [1]. Among systemic antipsoriatic drugs, cyclosporine (Cys-A) is known to be highly effective in the treatment of psoriasis in adults, while data on paediatric use are scarce. In this study, we aimed to expand the current knowledge of the use of Cys-A in paediatric psoriasis by assessing its effectiveness and safety in 38 children with moderate to severe plaque psoriasis. 


\section{Patients and Methods}

\section{Study design and study population}

A retrospective cohort study was conducted to identify case records of all consecutive patients under 17 years of age who were given cyclosporine for moderate to severe plaque psoriasis from 2010 until 2014. The setting was a number of Italian dermatology centers belonging to the network of "Pediatric Dermatology Group" of the Italian Society of Dermatology (SIDeMaST). Patients were identified through databases or registries. The following information was collected: gender, age, age at onset of psoriasis, association with psoriatic arthritis and other comorbidities, age at onset of CysA treatment, age at onset of previous systemic treatments, psoriasis severity assessment using psoriasis area severity index (PASI) before each treatment and after 12-16 weeks of treatment, duration treatment, reasons for withdrawal or switch, physician's notes.

Eligible for this study were considered only patients $<17$ years who had all requested data available and who had not concomitant systemic antipsoriatic treatment while they were taking Cys-A. At first commencement, all patients had baseline screening for full blood count, electrolytes, creatinine, liver function tests and urine analysis. Safety was assessed by using the results of vital signs, physical examination, laboratory tests and physician and patient evaluation of adverse events according to single center protocols. The efficacy was assessed by the proportion of subjects who achieved $\geq 75 \%$ reduction in baseline PASI score (PASI 75) after 16 weeks of therapy. Concomitant treatment, either with topical steroids, vitamin D3 ointments, coal tar preparations or anthralin, was possible. Descriptive statistics (eg, percentage, means) were used to summarize the data. 


\section{Results}

\section{Patient characteristics}

We identified a total of 56 potential subjects younger than 17 years with psoriasis who were treated with Cys-A between January 1, 2010, and December 31, 2014. After screening the complete medical records from all health care providers, 38 patients with plaque psoriasis fulfilled criteria for inclusion in the incidence cohort. 18 excluded subjects had diagnoses other than plaque psoriasis (eg, pustular, erythrodermic or guttate psoriasis). The patients, thirteen males and twenty-five females, were aged 1-17 years (mean age 8.7 years) at diagnosis of psoriasis. A positive familial history of psoriasis was found in seventeen patients (44.7\%). In particular ten patients had family history of psoriasis from the paternal side, six from the maternal line, one patient 1 from both parental sides. Medical histories included arthritis in two patients, history of streptococcal recurrent pharyngotonsillitis in five patients, obesity (as defined according to World Health Organization) in three patients, psychomotor delay in one patient, allergic rhinitis in one patient, gastro-esophageal reflux in one patient. No history of diabetes, hypertension, Crohn disease, celiac disease and irritable bowel syndrome was recorded in any children. Twenty-nine patients had no medical problems. All patients were commenced on Cys-A for moderate to severe psoriasis recalcitrant to topical treatment and/or narrowband ultraviolet B (nbUVB) or other systemic treatments. All patients had received topical treatment before. Eighteen patients (47.3\%) had previously been treated with nbUVB. One patients had previously been treated with methotrexate, one with etanercept, two with acitretin, four with psoralen UVA. Thus in 30 patients (78.9\%) Cys-A had been administered as first line systemic treatment. At commencement of Cys-A, patients had a mean age of 12.3 years (range 5-17).

\section{Treatment duration and dosage}

Cys-A was commenced at 2.1-3.5 $\mathrm{mg} / \mathrm{kg} / \mathrm{day}$ and given in two separate doses each day as oral solution or capsules according to the treating physician's and patients' preferences. An incremental increase up to a maximum daily dosage of $5 \mathrm{mg} / \mathrm{kg} / \mathrm{die}$ was occasionally made based on clinical 
response. Once the disease was controlled and stable, the dose might be tapered gradually according to clinical response. The median maintenance dosage per day was $3.2 \mathrm{mg} / \mathrm{kg}$ (range 2-5 $\mathrm{mg} / \mathrm{kg}$ ). The mean total duration of the first treatment cycle was 5.7 months (range 1-24 months) (Table 1).

\section{Effectiveness, tolerability and safety.}

Fifteen patients (39.47\%) were categorized as responders to Cys-A. Four out of them were able to stop cyclosporine for intervals of between two and six months, before requiring re-introduction of the treatment. Response rate did not change if it was calculated only in first time users of Cys-A. In this case there were twelve responders out from thirty patients (40\%). 8 patients discontinued cyclosporine due to side effects. In particular, an increase in serum creatinine level of $>30 \%$ was reported after three months of therapy in one patient; an increase of hypertriglyceridaemia in another patient. Two patients experienced headache, two gastro-intestinal disturbances, one recurrent bacterial superficial skin infections (pyodermitis), one hypertrichosis. All side effects resolved after stopping treatment. Fifteen patients (39.47\%) were non responders. Twelve of them were commenced on other systemic therapies. 


\section{Discussion}

Onset of psoriasis before the third or fourth decade of life is associated with greater likelihood of family history of the disease, increased severity of disease, and psychological morbidity [5]. In earlyonset psoriasis carriage of HLA-Cw6 and environmental triggers, such as $\beta$-haemolytic streptococcal infections, are considered main factors of disease expression [6]. Psoriasis in childhood and adolescence can cause a severe emotional burden and impair the health-related quality of life. Children and adolescents with psoriasis are more likely to experience anxiety, depression, decreased sexual intimacy, joint pain, chronic itching, and decreased perception of social connectivity [7-9]. Affected children are also significantly more at risk of developing psychiatric disorders [10].

Treatment of moderate to severe psoriasis in childhood is often challenging. According to expert consensus systemic therapeutic agents should be considered in cases of inadequate response to, contraindications towards topical or UV therapy as well as in extensive and chronic courses [11]. However studies on children are insufficiently documented with regard to dosing, efficacy, and safety. Consequently, most antipsoriatic systemic therapies are not licensed for paediatric use. Anyway physicians rely on experience from the application of these drugs for adults or other pediatric diseases [12]. Doses for children are often derived by scaling from adult dosages after adjusting for body weight. Until March 2015, etanercept was the only systemic drug licensed for plaque psoriasis in children aged six years or older [13]. Etanercept has grade A recommendation in two systematic evidence-based updates [14-15]. According to European Medicine Agency, etanercept should be used for the treatment of chronic severe plaque psoriasis in children and adolescents who are inadequately controlled by, or are intolerant to, other systemic therapies or phototherapies. Since April 2015 adalimumab has been approved by the European Commission or the treatment of severe chronic plaque psoriasis in children and adolescents from four years of age who have had an inadequate response to or are inappropriate candidates for topical therapy and phototherapies. 
Moreover, since June 2015 ustekinumab has been approved by the European Commission for the treatment of moderate-to-severe plaque psoriasis in adolescent patients from the age of 12 years and older, who are inadequately controlled by, or are intolerant to, other systemic therapies or phototherapies.

Consequently, etanercept, and ustekinumab should be administered following off-label use of other drugs, such as methotrexate, acitretin and Cys-A, since all these have not been registered for the treatment of childhood psoriasis. Adalimumab could be used as first choice systemic treatment, but only in severe form of the disease.

Cys-A is an immunosuppressant agent which produces calcium-dependent, specific, reversible inhibition of transcription of interleukin-2 and several other cytokines, most notably in T helper lymphocytes. As a result, the production of a range of cytokines is reduced [16]. Cys-A is approved for severe form of psoriasis and is included among conventional systemic treatments. It is considered an effective therapeutic option for therapy of moderate and severe psoriasis [17]. Adverse effects are common and usually dose-related. In addition with the well-known risk of increase of serum creatinine values and developing arterial hypertension, Gisondi et al reported significant increase of mean cholesterol and triglyceride levels also as short- term effect (up to 16 weeks) after starting Cys-A in a prospective cohort of adult patients [18]. The presence of comorbidities as diabetes, hypercholesterolaemia and hypertension and average baseline values of laboratory parameters can certainly intensify the risk of side effects. Therefore, the lowest possible maintenance dose and shortest treatment period are advisable. Cys-A is not registered for childhood psoriasis. The literature in children is limited, but extensive experience in children may be drawn from transplantation medicine and atopic dermatitis [11]. About clinical evidences published on childhood psoriasis, Cys-A proved to be effective for controlling resistant pediatric psoriasis in a recently published case series [19]. Lack of response (33\%) was similar to that of our group of patients (39.47\%). In their systematic literature reviews, De Yager et al and Van Geel et al [14-15] considered 4 studies with Cys-A embracing only 9 patients [20-23]. The Authors concluded that 
efficacy of Cys-A in childhood psoriasis is "ambiguous" since one case series of 4 young children affected with atypical forms of psoriasis (neonatal, acral, or immunodeficiency-associated) did not show any response [20]. Dutch guidelines duplicated this opinion [24]. Safety issues were sparsely described. Due to the use of antipsoriatic combination therapies and/or unclear outcome measure, other studies were excluded from the former mentioned systematic reviews [14-15]. Among these, there were the Perret' study reporting beneficial results in three children aged from 7 to 11 years [25] and the Pereira's case series reporting efficacy of Cys-A in five children with severe plaque psoriasis and one infant with pustular psoriasis [26]. The treatment was found to be well tolerated with no significant side-effects.

Therapeutic algorithms [14-15] propose methotrexate as the therapy of choice of childhood psoriasis if systemic treatment is need. According to a German consensus Cys-A may be justified only for selected children with severe flairs of psoriasis, when other therapeutic approaches have failed [11].

Our study represent the largest case series of pediatric patients treated with Cys-A. There was a distinct female preponderance, since the sex ratio of the group was $2.69: 1$ in favour of females. Gender difference may account for a higher frequency of psoriasis in girls than in boys in the age group under 20 years, in contrast to adults [11]. In our case series, Cys-A was administered as the first systemic therapy in a consistent quote of patients (78.9\%). A possible explanation for such attitude could be due to a broad and long lasting experience in managing this drug in adults. Cys-A has been proved to be the most frequently used systemic antipsoriatic therapy in Italy [27-28]. In our cohort of 38 children, Cys-A was effective in about $40 \%$ of patients and well tolerated. However, efficacy of Cys-A appears to be lower in childhood since PASI 75 is achieved at the end of induction therapy, i.e. after 12 weeks, in 50-70\% of adult patients [17]. Nevertheless children have higher BSA-to-weight ratios. They may present different pharmacokinetics for Cys-A and consequently require doses 
higher than the weight-adjusted doses [12,20]. Eight patients $(21.05 \%)$ discontinued the treatment due to transient laboratory anomalies or adverse events, although serious events were not identified. The limitations of our study pertains to its retrospective and multi-center setting with not-standardised treatment protocols. In addition, kidney function was not formally investigated.

\section{Conclusions}

Psoriasis often presents in childhood. Children with psoriasis commonly endorse negative QOL even in presence of mild disease. Thus treatment must be tailored according not only to patient age, extent and distribution of clinical lesions, but also to the impact on QOL. Individually planning and decisions shared together with the patients and their parents are essential to gain maximal safety, comfort and success.

Our study shows that Cys-A can be used in carefully selected and monitored patients and may represent an alternative treatment for severe flares of psoriasis. Definitely, biologics could expand the treatment repertoire and bridge a gap about the unmet need for treatment options in recalcitrant pediatric psoriasis. Health systems should implement registries or extensive databases to collect data on children and adolescents beginning systemic treatments and obtain prospective data on efficacy and safety profiles of old and new drugs. 


\section{References}

1. Benoit S, Hamm H. Childhood psoriasis. Clin Dermatol 2007; 25:555-62.

2. Augustin M, Glaeske G, Radtke MA et al. Epidemiology and comorbidity of psoriasis in children. Br J Dermatol 2010; 162:633-6.

3. Morris A, Rogers M, Fisher G, Williams K. Childhood psoriasis: a clinical review of 1262 cases. Pediatr Dermatol 2001; 18:188-198.

4. Huerta C, Rivero E, Rodriguez LA. Incidence and risk factors for psoriasis in the general population. Arch Dermatol 2007;143:1559-65.

5. Stuart P, Malick F, Nair RP et al. Analysis of phenotypic variation in psoriasis as a function of age at onset and family history Arch Dermatol Res. 2002; 294: 207-13.

6. Griffiths CE, Barker JN. Pathogenesis and clinical features of psoriasis. Lancet. 2007; 370: $263-7$.

7. de Jager ME, van de Kerkhof PC, de Jong EM, Seyger MM. A cross-sectional study using the Children's Dermatology Life Quality Index (CDLQI) in childhood psoriasis: negative effect on quality of life and moderate correlation of CDLQI with severity scores. Br J Dermatol 2010; 16:1099-101.

8. Ganemo A, Wahlgren CF, Svensson A. Quality of life and clinical features in Swedish children with psoriasis. Pediatr Dermatol 2011; 28:375-9.

9. de Jager ME, de Jong EM, van de Kerkhof PC et al. An intrapatient comparison of quality of life in psoriasis in childhood and adulthood. J Eur Acad Dermatol Venereol $2011 ; 25: 828-31$.

10. Kimball $A B, W u E Q$, Guerin $A$ et al. Risks of developing psychiatric disorders in pediatric patients with psoriasis. J Am Acad Dermatol 2012; 67: 651-7.

11. Sticherling M, Augustin M, Boehncke W-H et al. Therapy of psoriasis in childhood and adolescence -a German expert consensus. J Dtsch Dermatol Ges 2011; 9:815-23. 
12. Fotiadou C, Lazaridou E, Ioannides D. Management of psoriasis in adolescence. Adolesc Health Med Ther. 2014; 5:25-34.

13. Paller AS, Siegfried EC, Langley RG et al. Etanercept treatment for children and adolescents with plaque psoriasis. N Engl J Med 2008; 358: 241-51.

14. De Jager ME, de Jong EM, van de Kerkhof PC, Seyger MM. Efficacy and safety of treatments for childhood psoriasis: a systematic literature review. J Am Acad Dermatol 2010; 62: 1013-30.

15. van Geel MJ, Mul K, de Jager ME et al. Systemic treatments in paediatric psoriasis: a systematic evidence-based update. J Eur Acad Dermatol Venereol. 2015; 29:425-37.

16. Faulds D, Goa KL, Benfield P. Cyclosporin. A review of its pharmacodynamic and pharmacokinetic properties, and therapeutic use in immunoregulatory disorders. Drugs. 1993; 45: 953-1040.

17. Mrowietz U, Klein CE, Reich K et al. Cyclosporine therapy in dermatology. J Dtsch Dermatol Ges. 2009; 7: 474-9.

18. Gisondi, P., Cazzaniga, S., Chimenti, S et al. Metabolic abnormalities associated with initiation of systemic treatment for psoriasis: evidence from the Italian Psocare Registry. J Eur Acad Dermatol Venereol 2013 ; 27: 30-41.

19. Garber C, Creighton-Smith M, Sorensen EP et al. Systemic Treatment of Recalcitrant Pediatric Psoriasis: A Case Series and Literature Review. J Drugs Dermatol. 2015;14(8): 881-6.

20. Mahe E, Bodemer C, Pruszkowski A et al. Cyclosporine in childhood psoriasis. Arch Dermatol 2001; 137:1532-3.

21. Kilic SS, Hacimustafaoglu M, Celebi S et al. Low dose cyclosporin A treatment in generalized pustular psoriasis. Pediatr Dermatol 2001; 18: 246-8. 
22. Alli N, Gungor E, Karakayali $G$ et al. The use of cyclosporin in a child with generalized pustular psoriasis. Br J Dermatol 1998; 139:754-5.

23. Torchia D, Terranova M, Fabbri P. Photosensitive psoriasis in a vitiligo patient. J Dermatol 2006;33:880-3.

24. Zweegers J, de Jong EM, Nijsten TE et al. Summary of the Dutch S3-Guidelines on

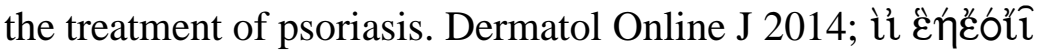

25. Pereira, T., Vieira, A., Fernandes, J, Sousa-Basto, A. Cyclosporin A treatment in severe childhood psoriasis. J Eur Acad Dermatol 2006; 20: 651-6.

26. Perret CM, Ilchyshyn A, Berth-Jones J. Cyclosporin in childhood psoriasis. J Dermatolog Treat. 2003;14:113-8.

27. Naldi L, Griffiths Traditional therapies in the management of moderate to severe chronic plaque psoriasis: an assessment of the benefits and risks. Br J Dermatol 2005; 152: $597-615$

28. Altomare G, Ayala F, Bardazzi F et al. Italian Consensus Conference. Consensus on the use of cyclosporine in dermatological practice. Italian Consensus Conference. G Ital Dermatol Venereol 2014; 149: 607-25. 
Table 1 Overview of clinical characteristics, treatment course and outcome of 38 children and adolescent with plaque psoriasis treated with CysA.

\begin{tabular}{|l|l|}
\hline Number of patients & 38 \\
\hline Males & $13(34.2 \%)$ \\
\hline Females & $25(65.7 \%)$ \\
\hline Family history & $17(44.7 \%)$ \\
\hline Previous phototherapy & $18(47.3 \%)$ \\
\hline Previous systemic treatments & $8(21.05 \%)$ \\
\hline Age at start of CysA & $5-17$ years (median 12.3) \\
\hline Daily dose at start of CysA & $2.1-3.5 \mathrm{mg} / \mathrm{kg}$ \\
\hline Maintenance daily dose of CysA & $2-5$ mg/kg (median 3.2) \\
\hline Duration of treatment & $1-24$ months (median 5.7) \\
\hline Response & $15(39.47 \%)$ \\
\hline Adverse Events & $8(21.05 \%)$ \\
\hline
\end{tabular}

